\title{
1 Configuring fixed-coefficient active control systems for traffic noise reduction
}

2

3 Sipei Zhao ${ }^{\mathrm{a}}$, Xiaojun Qiu ${ }^{\mathrm{a}}$, Jordan Lacey ${ }^{\mathrm{b}}$, Simon Maisch $^{\mathrm{b}}$

$4 \quad{ }^{a}$ Centre for Audio, Acoustics and Vibration, Faculty of Engineering and IT, University of Technology Sydney, Australia

$5 \quad$ bIAL Sound Studios, RMIT University, Australia

6

7 Practical implementation of active noise control (ANC) systems for outdoor traffic noise reduction

8 remains rare. One challenge is the difficulty of configuring an ANC controller due to moving noise

9 sources, which are typically located far from ANC systems. In this paper, a pseudo noise source method

10 is proposed for configuring fixed-coefficient feedforward ANC systems for traffic noise control. First,

11 a minimum of one pseudo noise source is placed near an ANC system to determine the control

12 coefficients in the tuning stage. Second, the ANC systems are run to reduce the noise from far-field

13 traffic noise sources using the optimal control coefficients in the cancelling stage. The feasibility and

14 limitations of the proposed method are investigated by illustrating the effect of the pseudo noise source

15 position on the noise reduction performance of the ANC system. The simulation results show that the

16 performance of the ANC system increases with distance when the pseudo noise sources move farther

17 from the system but approaches a constant when the pseudo noise sources are in the far field. The indoor

18 experimental results are consistent with the simulation results. The outdoor experimental results of a

19 six-channel coupled system show a noise reduction of $3 \mathrm{~dB}$ below $500 \mathrm{~Hz}$ at the position of a dummy

20 head. 


\section{Introduction}

Traffic noise is generally random, non-stationary, broadband, and detected in large unconfined spaces, which render it hard to control [1]. Noise barriers have been extensively employed to reduce traffic noise from highways [2]. In addition to regular rigid barriers [3], various modifications have been proposed to improve the noise reduction performance of passive noise barriers [4]. For example, sound absorbing materials have been applied on barrier surfaces facing traffic [5]; a diffracting edge has been adopted on barrier tops to form T-shape barriers, Y-shape barriers, and barriers with quadratic diffuser tops [6], and rough surface barriers have been used to achieve diffusive reflection and wavetrapping effects that attenuate multiple reflections in parallel noise barriers [4,7]. Recently, a new type of noise barrier that consists of an array of isolated scatterers has been introduced to reduce transportation noise [8].

Despite their prevalence, the performance of noise barriers in the lower frequency range is limited due to the physical size of the barriers [9]. Active noise control (ANC) systems can be employed to control low-frequency traffic noise in different ways, i.e., by directly creating a quiet zone with an ANC system [10] or applying an ANC system on top of a passive noise barrier to form an active noise barrier (ANB) [11]. Many studies have been devoted to the direct application of ANC systems to create quiet zones. Guo et al. employed multiple control sources to create a quiet zone in a free space [12]. Wright and Vuksanovic utilized ANC systems to reduce environmental noise by creating an acoustic shadow of a certain angle with eight secondary sources and microphones in an anechoic room [13].

In contrast to these studies, where the control sources were placed in a linear array to reduce noise from a single primary noise at a fixed position, Zou et al. developed a virtual sound barrier (VSB) system, which uses an array of loudspeakers and microphones in a three-dimensional space to create a quiet zone surrounded by error microphones [14]. Similarly, Epain et al. employed 30 loudspeakers and microphones to create a quiet zone inside a sphere with a radius of $0.3 \mathrm{~m}$; their results show that broadband noise can be cancelled in a frequency range up to $500 \mathrm{~Hz}$ [15].

These systems have been effective in creating quiet zones in laboratory environments, where a minimum of one loudspeaker was used to mimic the primary noise sources and the ANC controller was adaptively adjusted throughout experiments. However, none of the previous studies have been applied to real outdoor traffic noise control. In practical applications for traffic noise, noise from moving vehicles is typically located far from the ANC systems; thus, the system cannot be adaptively adjusted due to the non-stationary signal and relatively low signal-to-noise ratio onsite.

In addition to these direct applications of pure active control methods, ANC systems have also been applied on top of passive noise barriers to enhance their noise reduction performance. In a $40 \mathrm{~m}$ prototype active soft edge ANB system along a noise barrier, Ohnishi et al. employed numerous singlechannel independent analogue feedback control modules to construct a multichannel ANB system and achieved 2-4 dB extra noise reduction in the $250 \mathrm{~Hz}$ and $500 \mathrm{~Hz}$ octave bands [16]. The problem with 
the feedback control system is that it suffers from the waterbed effect and stability issues [17]. To overcome these problems, Zou et al. proposed a decentralized feedforward control ANB system; their results show that the system works effectively with both predefined control filter parameters and adaptive control systems [18].

Feedforward ANC systems have also been utilized to reduce traffic noise transmission through ventilation windows [19]. Fully-coupled multichannel feedforward systems are complicated and computationally demanding; therefore, decentralized feedforward systems are often utilized in research at the cost of inferior performance [20]. To extend feedforward ANC systems to large-scale applications, different algorithms have been explored to optimize the computational load and performance in fully-coupled and decentralized feedforward ANC systems [21].

Unfortunately, all of the above studies focused on a single fixed noise source case, which does not reflect the actual traffic noise scenario, where multiple moving noise sources are simultaneously present. Multiple moving noise sources hinder the application of active control systems. Uesaka et al. showed that the performance of a six-channel ANC system degraded when the noise source was mobile [22]. Omoto et al. also demonstrated that their adaptive multichannel ANC systems exhibited inferior performance for a moving noise source compared with a fixed noise source [23]. In practical applications of ANC systems in traffic noise reduction, the moving noise sources to be controlled are usually far from the ANC systems; hence, fixed noise sources do not exist for tuning the controller to obtain optimal coefficients.

This study is devoted to investigating the applications of fixed-coefficient feedforward ANC systems in actual traffic noise scenarios. This work is part of a research project on motorway noise management that combines cancellation and transformational methods to design an aesthetically pleasing soundscape in parklands near highways. This research focuses on the cancellation aspect; the transformation system was reported in Ref. [24].

The advantages of adopting fixed-coefficient feedforward ANC systems are their low cost and robustness. However, the application of adaptive multichannel systems on noise barriers, the length of which can be hundreds of meters, to control traffic noise remains impractical. To configure a fixedcoefficient ANC system, a minimum of one pseudo noise source is placed near the ANC system to set up the control coefficients in the tuning stage. After the controller is configured for this situation, the control coefficients are fixed, and the ANC system is utilized to cancel the actual noise from far-field moving noise sources. The performance of the proposed method is numerically and experimentally investigated. The paper is structured as follows: Section 2 formulates the theoretical equations, and Section 3 presents the simulation results for both single-channel systems and multichannel systems. The indoor experiments of single- and multichannel systems, as well as outdoor experiments of a sixchannel system with one reference microphone, are presented in Section 4. The limitations of this study is discussed in Section 5, and the conclusions are drawn in Section 6. 


\section{Theory}

This section introduces the fundamental theory and equations for the simulations performed to investigate the performance of the proposed method. For a multiple-reference multichannel ANC system, the total sound pressure at the error microphones is the sum of the primary noise and the control sound, namely, [25]

$$
\mathbf{e}(\omega)=\mathbf{p}(\omega)+\mathbf{Z}(\omega) \mathbf{X}(\omega) \mathbf{q}(\omega),
$$

where $\mathbf{p}(\omega)=\left[p_{1}(\omega), p_{2}(\omega), \ldots, p_{L}(\omega)\right]^{\mathrm{T}}$ and $\mathbf{e}(\omega)=\left[e_{1}(\omega), e_{2}(\omega), \ldots, e_{L}(\omega)\right]^{\mathrm{T}}$ denote the primary noise and the total sound pressure, respectively, at the error microphones. $L$ is the total number of error microphones, and $\mathbf{Z}(\omega)$ is an $L \times L$ matrix of the transfer functions from the $L$ control sources to the $L$ error microphones, as illustrated in Fig. 1(a). $\mathbf{X}(\omega)$ is a diagonal matrix with signals from the reference microphones, and $\mathbf{q}(\omega)$ represents the control coefficients. For the sake of brevity and clarity, the frequency dependency $(\omega)$ is omitted in the following context.

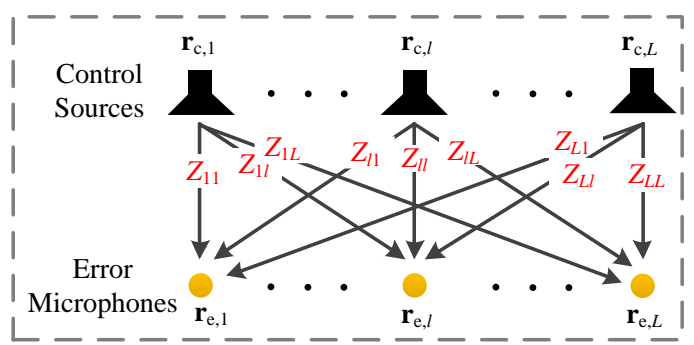

(a)

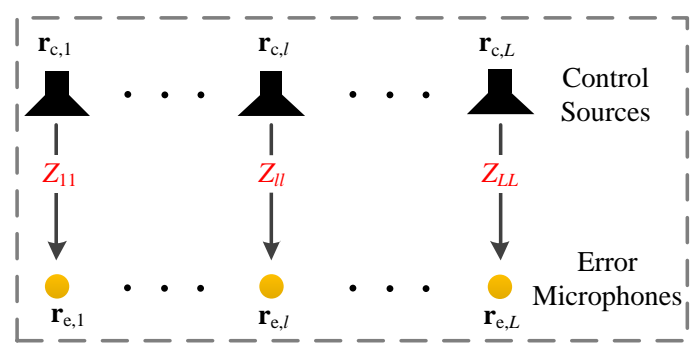

(b)

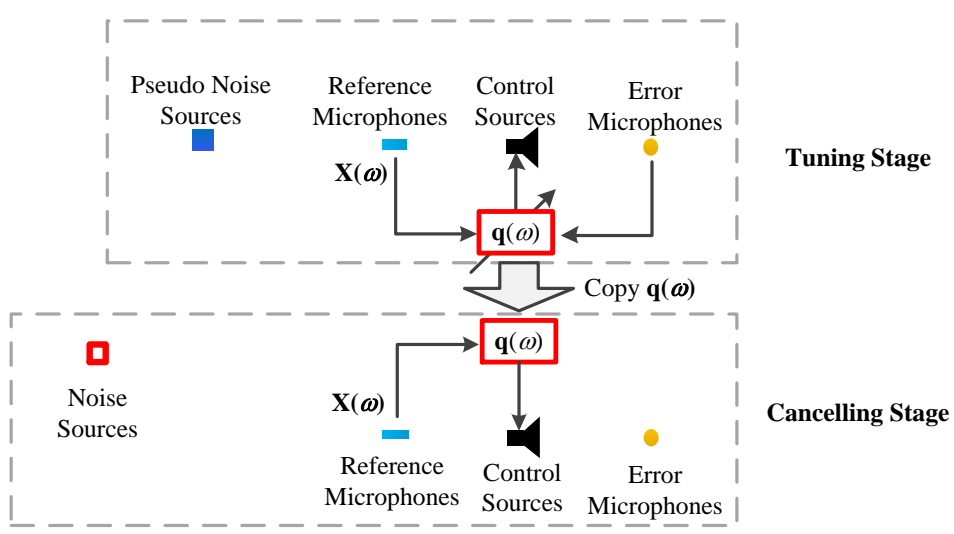

(c)

Fig. 1. Illustration of (a) the definition of the transfer function matrix $\mathbf{Z}$ for the coupled system, (b) the definition of the transfer function matrix $\mathbf{Z}_{0}$ for the multiple single-channel system, and (c) the block diagram of the proposed method.

For most noise control applications in large spaces, multiple channels must be employed; however, implementation with a fully-coupled, multiple-reference, multichannel ANC system is difficult as the computational complexity rapidly increases with the number of channels. Therefore, two simplified 
systems are investigated in this paper. The first system is an ANB system that has a passive barrier that can have a length of hundreds of meters, and the second system is designed to create a small quiet area.

The first system consists of multiple single-channel ANC modules, where the control output of each module is solely determined by the corresponding reference and error signals. The cost function can be defined as the squared sound pressure at each error microphone [26],

$$
J_{l}=e_{l}{ }^{*} e_{l}+\beta_{l} q_{l}{ }^{*} q_{l},
$$

where the superscript * denotes the complex conjugate, $\beta_{l}$ is a regularization factor, and the subscript $l$ $=1,2, \ldots, L$ denotes the $l$-th channel. The optimal control coefficients can be obtained by minimizing Eq. (2) as [26]

$$
\mathbf{q}=-\left[\left(\mathbf{Z}_{0} \mathbf{X}\right)^{\mathrm{H}}\left(\mathbf{Z}_{0} \mathbf{X}\right)+\boldsymbol{\beta} \mathbf{I}\right]^{-1}\left(\mathbf{Z}_{0} \mathbf{X}\right)^{\mathrm{H}} \mathbf{p}
$$

where the superscript ${ }^{\mathrm{H}}$ denotes the Hermitian transpose, $\mathbf{I}$ is the identity matrix, $\boldsymbol{\beta}=\operatorname{diag}\left(\beta_{1}, \ldots, \beta_{l} \ldots\right.$, $\beta_{L}$ ) is the diagonal matrix of the regularization factors, and $\mathbf{Z}_{0}$ is an $L \times L$ matrix for which the diagonal elements are identical to $\mathbf{Z}$ while the off-diagonal elements are zero, as illustrated in Fig. 1(b). As standardized single-channel modules are easily mass-produced, the extension of such a system to practical noise barriers with a length of hundreds of meters is possible. However, the performance of this system may not be optimal as the contributions from the other control sources are not considered when optimizing the control coefficients.

The second system is a coupled multichannel ANC system with one reference microphone, and the cost function is defined as the sum of the squared sound pressure at all error microphones,

$$
J=\mathbf{e}^{\mathrm{H}} \mathbf{e}+\beta \mathbf{q}^{\mathrm{H}} \mathbf{q}
$$

where $\beta$ is a regularization factor. The optimal control coefficients for the coupled multichannel ANC system can be obtained as [26]

$$
\mathbf{q}=-\left[(\mathbf{Z} x)^{\mathrm{H}}(\mathbf{Z} x)+\beta \mathbf{I}\right]^{-1}(\mathbf{Z} x)^{\mathrm{H}} \mathbf{p},
$$

where $x$ is the sound pressure at the reference microphone.

In the traffic noise control scenario, the moving noise sources to be controlled are typically located far from the ANC system and fixed noise sources do not exist to update the controller. To solve this problem, one or multiple pseudo noise sources is utilized to set up the optimal control coefficients. A diagram of the proposed method is illustrated in Fig. 1(c). In the tuning stage, which is shown at top of the diagram, pseudo noise sources with random noise signals are placed near the ANC system to tune the control filter coefficients. After the optimal control filter coefficients are obtained from the tuning, they are fixed to the controller. The controller does not update when the coefficients are used to cancel the far-field noise in the cancelling stage, as shown on the bottom of the diagram. The effect of the pseudo noise source position on the performance of ANC systems designed with the proposed method is investigated in this study. 
In the tuning stage, a pseudo noise source at position $\mathbf{r}_{\mathrm{m}}=\left(x_{\mathrm{m}}, y_{\mathrm{m}}, z_{\mathrm{m}}\right)$, as depicted by the blue squares in Fig. 2(a), is used to obtain the control coefficients; thus, for systems that consist of multiple single-channel modules,

$$
\mathbf{q}_{\mathrm{m}}=-\left[\mathbf{Z}_{0} \mathbf{X}_{\mathrm{m}}\right]^{-1} \mathbf{p}_{\mathrm{m}},
$$

where $\mathbf{p}_{\mathrm{m}}$ and $\mathbf{X}_{\mathrm{m}}$ denote the sound pressures received at the error microphone and the reference microphone, respectively, from the pseudo noise source. When multiple pseudo noise sources are employed, the locations are denoted by $\mathbf{r}_{\mathrm{m}, 1}, \ldots, \mathbf{r}_{\mathrm{m}, u}, \ldots, \mathbf{r}_{\mathrm{m}, U}$, where $U$ is the total number of pseudo noise sources. The regularization factor is assumed to be 0 for the best performance. The objectives of the simulations are to investigate the feasibility of the proposed method and to examine the best possible performance. The proposed method is for fixed-coefficient ANC systems; thus, the robustness is not considered in this study. In the experiments, a leakage factor was applied by the Antysound Tiger ANCII controller to increase the robustness of the adaptive algorithm when adjusting the control filter coefficients for the pseudo noise sources. A leakage factor is equivalent to a regularization factor, which increases the stability of the ANC system at the cost of a decrease in noise reduction performance [27].

For the coupled multichannel system with one reference microphone,

$$
\mathbf{q}_{\mathrm{m}}=-\left[\mathbf{Z} x_{\mathrm{m}}\right]^{-1} \mathbf{p}_{\mathrm{m}}
$$

where $\mathbf{p}_{\mathrm{m}}$ and $x_{\mathrm{m}}$ denote the pseudo noise source sound pressures received at the error microphone position and reference microphone position, respectively.

Substituting the optimal control coefficients in Eqs. (6) into Eq. (1), and then substituting Eq. (7) into Eq. (1), the total sound pressure at the error microphones for far-field noise at position $\mathbf{r}_{\mathrm{n}, v}(v=1$, $2, \ldots, V$, where $V$ is the total number of primary noise sources, which are depicted by the red squares in Fig. 2) can be expressed as

$$
\mathbf{e}_{\mathrm{n}}=\mathbf{p}_{\mathrm{n}}-\mathbf{Z} \mathbf{X}_{\mathrm{n}}\left[\mathbf{Z}_{0} \mathbf{X}_{\mathrm{m}}\right]^{-1} \mathbf{p}_{\mathrm{m}}
$$

for the system that consists of multiple single-channel modules and

$$
\mathbf{e}_{\mathrm{n}}=\mathbf{p}_{\mathrm{n}}-\frac{x_{\mathrm{n}}}{x_{\mathrm{m}}} \mathbf{p}_{\mathrm{m}}
$$

for the coupled multichannel ANC system with one reference microphone, respectively.

Noise reduction (NR) at the error microphone locations is defined as

$$
N R=10 \log _{10}\left(\frac{\mathbf{p}_{\mathrm{n}}^{\mathrm{H}} \mathbf{p}_{\mathrm{n}}}{\mathbf{e}_{\mathrm{n}}^{\mathrm{H}} \mathbf{e}_{\mathrm{n}}}\right) .
$$

The proposed method is verified with a single-channel ANC system, and then the performance of the two systems is investigated by numerical simulations and experiments. Note that the acoustic feedback from the control source to its reference microphone may affect the stability of each single-channel ANC system. Many methods have been explored to solve this issue [27] but they are not considered in this study. 


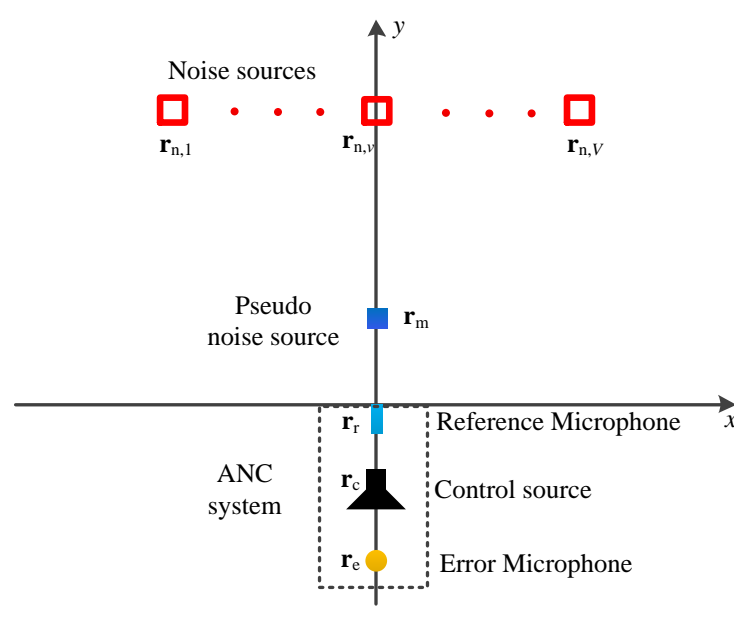

(a)

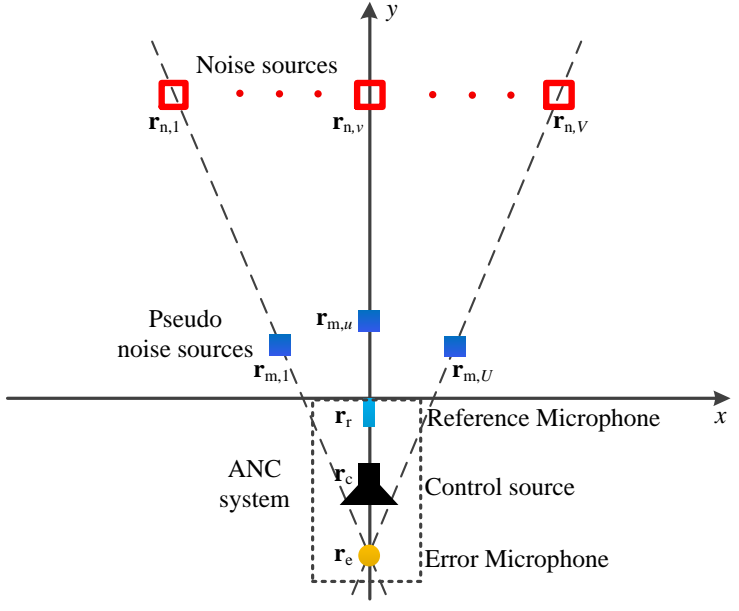

(b)

Fig. 2. Diagram of a single-channel ANC system configured with (a) one pseudo noise source and (b) three pseudo noise sources.

\section{Simulations}

The numerical simulations were performed in MATLAB R2017a. The three-dimensional free field Green function $Z_{i j}=\frac{\mathrm{e}^{-\mathrm{j} k\left|\mathbf{r}_{i}-\mathbf{r}_{j}\right|}}{4 \pi\left|\mathbf{r}_{i}-\mathbf{r}_{j}\right|}$, where $k$ is the wavenumber, and $\mathbf{r}_{i}$ and $\mathbf{r}_{j}$ are the coordinates of the $i$-th sound source and the $j$-th receiver, respectively, was employed in the transfer matrix $\mathbf{Z}$ [27].

\subsection{Single-channel systems}

A single-channel system was investigated as it can be implemented as a low-cost device to create a small quiet zone along a noisy traffic road. The control coefficients of the single-channel ANC system can be determined using a minimum of one pseudo noise sources, as shown in Fig. 2. In the simulations for the single-channel system, one pseudo noise source $(U=1)$ and three pseudo noise sources $(U=3)$ were employed, while 13 far-field noise sources $(V=13)$ were utilized.

In the simulations, the reference microphone location is set as the origin of the coordinate system, as shown in Fig. 2. The control source and the error microphone are located $0.15 \mathrm{~m}$ and $0.3 \mathrm{~m}$, respectively, from the reference microphone in the negative $y$ direction. In practical traffic noise situations, many incoherent noise sources exist along a motorway [28]. To simulate this situation, 13 random-phased monopole sources evenly distributed along a line of $60 \mathrm{~m}$ were employed, and the pseudo noise source was placed at numerous positions to investigate the noise reduction performance. The length of the incoherent primary noise sources $(60 \mathrm{~m})$ was selected based on an estimation from outdoor experiments conducted in a park near a motorway in Richmond, Victoria, Australia.

When a single pseudo noise source is utilized, as shown in Fig. 2(a), the simulation results are shown in Fig. 3(a) at different frequencies, where the vertical bars indicate the standard deviation of 100 trials. The abscissa in Fig. 3 is the dimensionless number $k y_{\mathrm{m}} L_{0} / d_{0}$, where $k$ is the wavenumber, $y_{\mathrm{m}}$ 
is the distance between the pseudo noise sources and the ANC system, $L_{0}$ is the length of the incoherent

212 primary noise sources, and $d_{0}$ is the distance between the primary noise sources and the ANC system.

213 In Fig. 3(a), both $L_{0}$ and $d_{0}$ are $60 \mathrm{~m}$, whereas in Fig. 3(b), $d_{0}=60 \mathrm{~m}$ and $L_{0}=60 \mathrm{~m}, 120 \mathrm{~m}$, and $240 \mathrm{~m}$.

214 In the simulations, $y_{\mathrm{m}}$ was varied from $0.1 \mathrm{~m}$ to $100 \mathrm{~m}$.

215 Noise reduction approaches a constant when the dimensionless number $k y_{\mathrm{m}} L_{0} / d_{0}$ is larger than $10 \pi$, regardless of the frequency (Fig. 3). This finding corresponds to the far-field condition $k y_{\mathrm{m}} \gg \pi$ in Fig. 3(a), where $L_{0} / d_{0}=1$. Therefore, the results conclude that the $N R$ increases with distance between the pseudo noise sources and the ANC system and approaches a constant when the pseudo noise sources are placed in the far field from the ANC system, i.e., $k y_{\mathrm{m}} L_{0} / d_{0}>10 \pi$. When the pseudo noise sources are placed in the far field, the sound pressure at the ANC system can be approximated by plane waves, which is similar to that from primary noise sources.

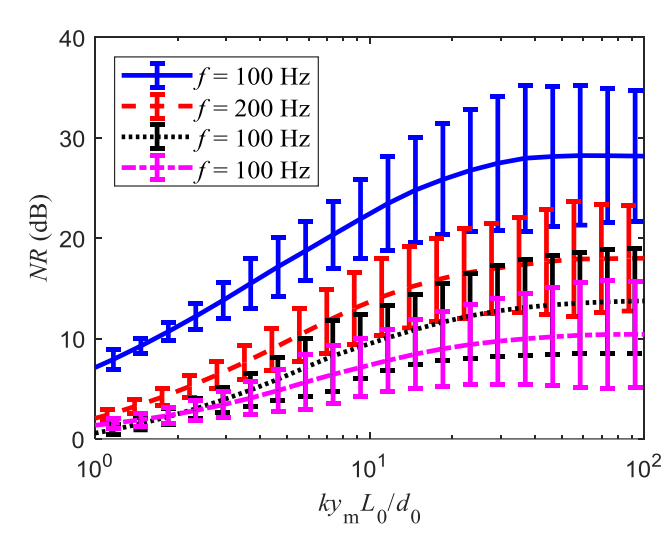

(a)

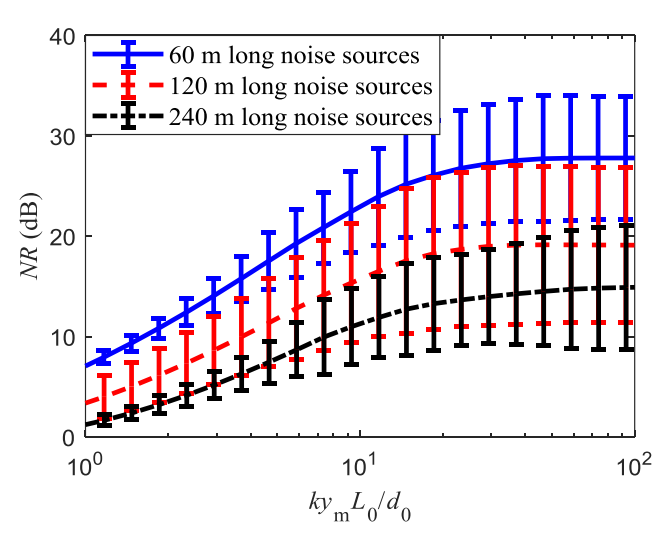

(b)

Fig. 3. NR (dB) as a function of the dimensionless number $k y_{\mathrm{m}} L_{0} / d_{0}$. (a) NR at different lengths of primary noise sources.

In practical applications, the actual noise sources on a motorway may exceed $60 \mathrm{~m}$. The simulated $\mathrm{NR}$ at $100 \mathrm{~Hz}$ when the length of the incoherent primary noise sources is $120 \mathrm{~m}$ and $240 \mathrm{~m}$ are illustrated in Fig. 3(b). NR approaches a constant when the dimensionless number $k y_{\mathrm{m}} L_{0} / d_{0}$ exceeds $10 \pi$, which coincides with the results in Fig. 3(a). In addition, the performance of the ANC system deteriorates when the length of the primary noise sources exceeds $60 \mathrm{~m}$. The maximum NR at $100 \mathrm{~Hz}$ for primary noise sources with lengths of $120 \mathrm{~m}$ and $240 \mathrm{~m}$ is $20 \mathrm{~dB}$ and $15 \mathrm{~dB}$, respectively, which is lower than that for $60 \mathrm{~m}$ noise sources ( $28 \mathrm{~dB}$ ). Figure 3 shows that the noise reduction performance of the singlechannel system decreases with increasing frequency. This finding is clearly illustrated in Fig. 5 for NR as a function of frequency.

The deviation of NR is large in Fig. 3 as the phases of the 13 incoherent noise sources were random for each run in the simulations. NR depends on the position of the pseudo noise source, the locations of 
the far-field noise sources, and the amplitudes and phases of the far-field noise sources. In the simulations, the locations of the far-field noise sources were fixed, and the amplitudes of all noise sources were assumed to be equal. For each pseudo source position, 100 trials of random phases of farfield noise sources were simulated, and the standard deviations are depicted by the vertical bars in Fig. 3.

The NR for the pseudo noise sources that are not on the $y$-axis are shown in Fig. 4 for $100 \mathrm{~Hz}, 300$ $\mathrm{Hz}, 500 \mathrm{~Hz}$, and $1000 \mathrm{~Hz}$, where the red squares indicate real noise source locations. In Fig. 4, each pixel corresponds to a pseudo noise source position and the colour denotes the NR value. For example, in Fig. 4(a), the NR for the pseudo noise source position at $x_{\mathrm{m}}=0$ and $y_{\mathrm{m}}=20 \mathrm{~m}$ is $28 \mathrm{~dB}$ (yellow), while the NR for the pseudo noise source position at $x_{\mathrm{m}}=20 \mathrm{~m}$ and $y_{\mathrm{m}}=10 \mathrm{~m}$ is $11 \mathrm{~dB}$ (blue). Therefore, the performance of the ANC system is sensitive to the position of the pseudo noise source, as shown in Figs. 3 and 4. The colour bar in Fig. 4 is fixed between $0 \mathrm{~dB}$ and $40 \mathrm{~dB}$ for the sake of clarity.

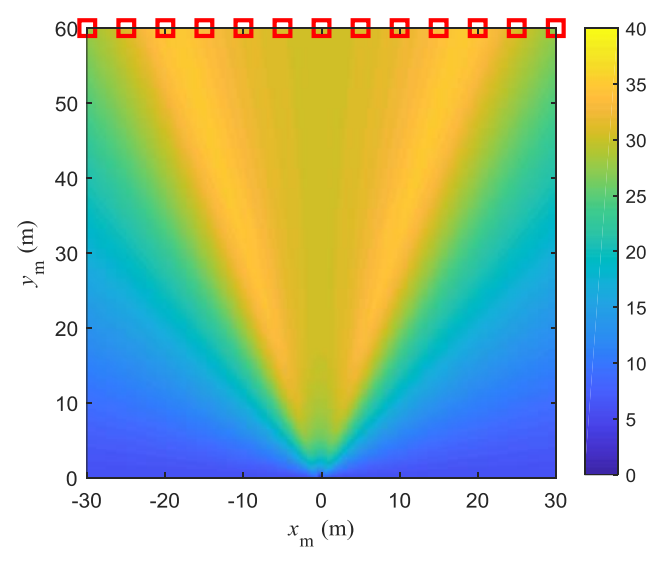

(a)

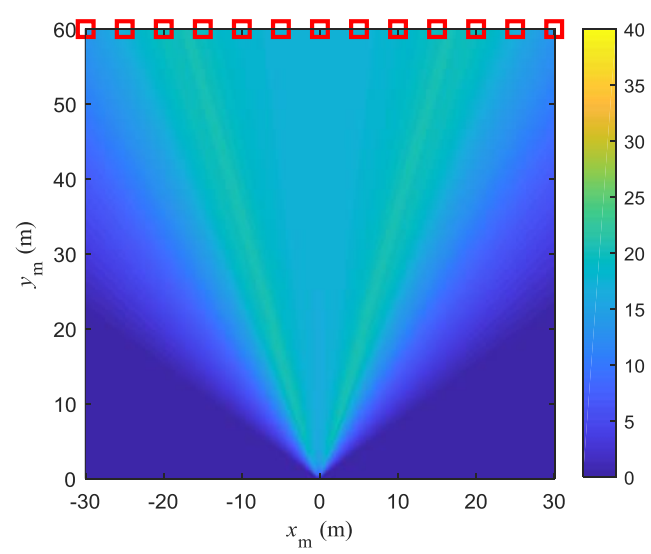

(c)

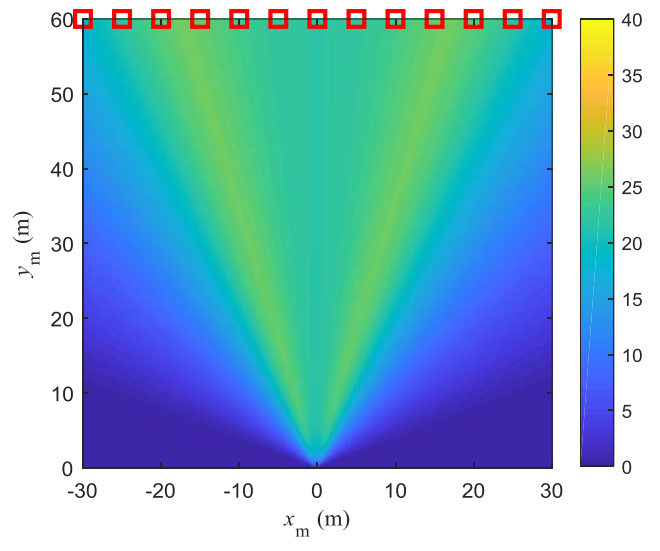

(b)

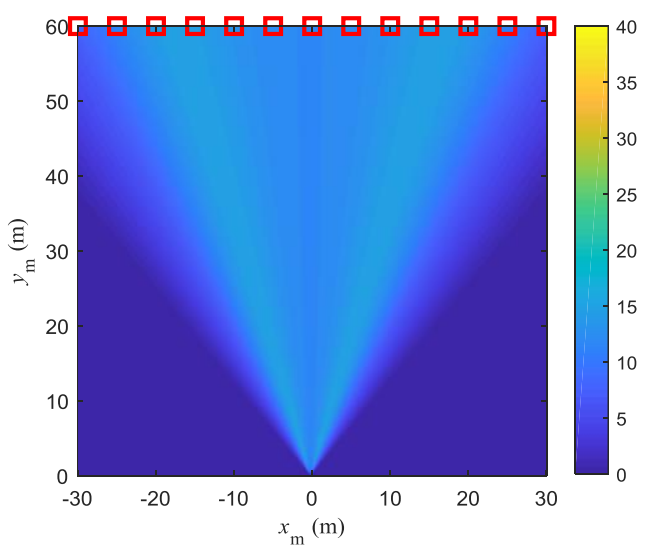

(d)

Fig. 4. Average $N R(\mathrm{~dB})$ of 100 trials for various single pseudo noise source positions when the channel ANC system, (a) $100 \mathrm{~Hz}$, (b) $300 \mathrm{~Hz}$, (c) $500 \mathrm{~Hz}$, and (d) $1000 \mathrm{~Hz}$ (red squares denote the 
When three pseudo noise sources are employed, as shown in Fig. 2(b), the pseudo noise sources are bounded by the angle formed between the 13 point sources and the error microphone to mimic the noise from the primary noise source. The pseudo noise sources can also be placed in a linear arrangement. However, an arc arrangement is more compact for mimicking noise from different directions. All three pseudo noise sources are located at the same distance from the reference microphone. The simulation results for three pseudo noise sources are compared with those for a single pseudo noise source in Fig. 5 , where the pseudo noise sources are placed $1 \mathrm{~m}$ and $20 \mathrm{~m}$ from the ANC system, respectively. The performance of the single-channel ANC system for both distances decreases with increasing frequency (Fig. 5), and configurations that employ more pseudo noise sources to simulate the noise from different directions slightly increase the NR over the entire frequency range from $100 \mathrm{~Hz}$ to $1000 \mathrm{~Hz}$. When the noise originates from a $60 \mathrm{~m}$ line of incoherent noise sources located $60 \mathrm{~m}$ from the ANC system, the highest NR is approximately $31 \mathrm{~dB}$ at $100 \mathrm{~Hz}$ and $12 \mathrm{~dB}$ at $1000 \mathrm{~Hz}$, which can be achieved by placing the pseudo noise sources $20 \mathrm{~m}$ from the ANC system. By employing five pseudo noise sources, the average NR can be improved by a maximum of $3 \mathrm{~dB}$, as shown in Fig. 5(b).

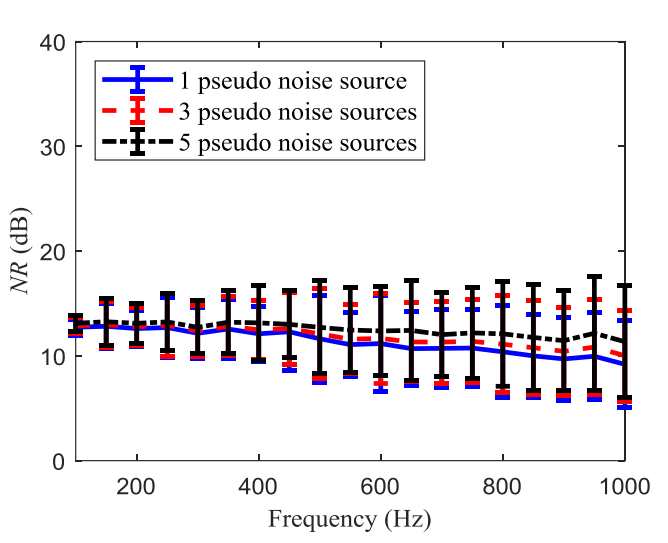

(a)

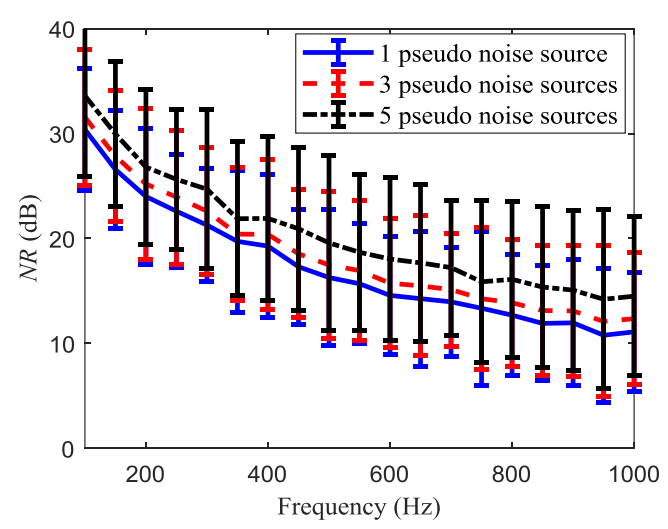

(b)

Fig. 5. NR (dB) as a function of the frequency for different numbers of pseudo noise sources when the pseudo noise sources are (a) $1 \mathrm{~m}$ and (b) $20 \mathrm{~m}$ from the single-channel ANC system. The noise originates from a $60 \mathrm{~m}$ line of incoherent sources located $60 \mathrm{~m}$ from the ANC system.

The proposed pseudo noise source method is feasible for configuring single-channel ANC systems to reduce the noise from a line of incoherent sources in the far field. The performance depends on the specific configurations. An average NR of more than $10 \mathrm{~dB}$ can be achieved at the error sensors at 1000 Hz. When the noise comes originates from a line of incoherent point sources far from the ANC system, moving the pseudo noise sources farther away can effectively increase the noise reduction. NR increases with the distance between the pseudo noise sources and the ANC system and then approaches a constant when the distance exceeds a critical value, which can be determined by $k y_{\mathrm{m}} L_{0} / d_{0}>10 \pi$. Using additional 
pseudo noise sources to simulate the noise from the directions of the actual noise sources can improve the noise reduction over the entire frequency range.

Note that these studies are based on numerical simulations. A theoretical formulation for NR dependence on distance and frequency is possible for a single-channel ANC system with one noise source, which is detailed in the Appendix. For a multichannel system, however, a simple theoretical formulation to predict the variability of NR with distance and frequency is difficult due to complications from multiple secondary sources.

\subsection{Multichannel systems}

Two simplified multichannel ANC systems were investigated, as shown in Fig. 6. Fig. 6(a) shows a multiple single-channel ANC system; its cost function is defined in Eq. (2). Fig. 6(b) shows a coupled multichannel ANC system with one reference microphone; its cost function is defined in Eq. (4). Only one reference microphone is used in the coupled multichannel ANC system in Fig. 6(b), as the fullycoupled multichannel ANC system with multiple reference microphones is computationally demanding and implementation in experiments is difficult.

In the simulations, a three-channel system $(L=3)$ was investigated. The coordinates of the reference microphones, control sources and error microphones in Fig. 6(a) are summarized in Table 1. For the coupled three-channel ANC system with one reference microphone in Fig. 6(b), the coordinates of the control sources and error microphones are equivalent, as shown in Table 1 but only one reference microphone at $(0,0,0)$ was employed.

Table 1. Coordinates of the reference microphones, control sources, and error microphones in the simulations and indoor experiments for the three-channel systems.

\begin{tabular}{|c|c|c|c|}
\hline \multirow{2}{*}{ Coordinates in meters } & \multicolumn{3}{|c|}{ Channel index $l=1,2, \ldots, L(L=3)$} \\
\cline { 2 - 4 } & $l=1$ & $l=2$ & $l=3$ \\
\hline Reference microphones $\mathbf{r}_{\mathrm{r}, l}=\left(x_{\mathrm{r}, l}, y_{\mathrm{r}, l}, z_{\mathrm{r}, l}\right)$ & $(-0.078,-0.010,0)$ & $(0,0,0)$ & $(0.078,-0.010,0)$ \\
\hline Control sources $\mathbf{r}_{\mathrm{c}, l}=\left(x_{\mathrm{c}, l}, y_{\mathrm{c}, l}, z_{\mathrm{c}, l}\right)$ & $(-0.039,-0.155,0)$ & $(0,-0.150,0)$ & $(0.039,-0.155,0)$ \\
\hline Error microphones $\mathbf{r}_{\mathrm{e}, l}=\left(x_{\mathrm{e}, l}, y_{\mathrm{c}, l}, z_{\mathrm{e}, l}\right)$ & $(-0.013,-0.252,0)$ & $(0,-0.250,0)$ & $(0.013,-0.252,0)$ \\
\hline
\end{tabular}




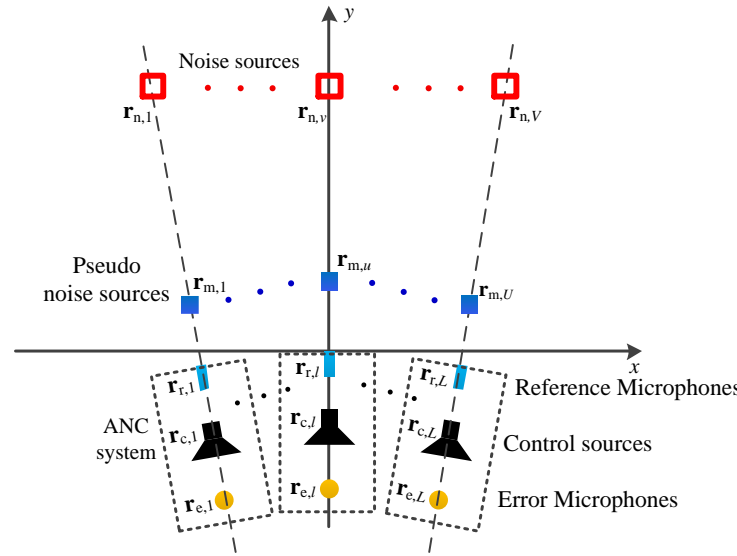

(a)

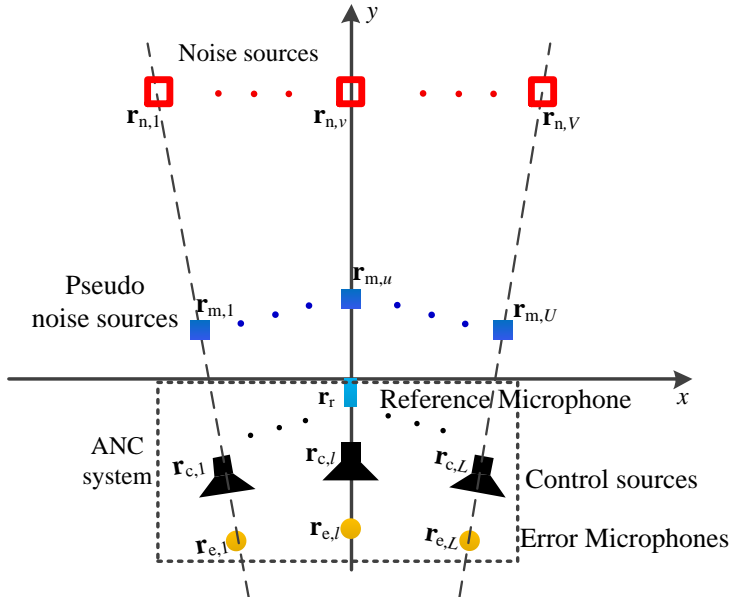

(b)

Fig. 6. Diagram of (a) a multiple single-channel ANC system and (b) a coupled multichannel

ANC system with one reference microphone.

The pseudo noise sources are placed along an arc to mimic the primary noises from different directions. A linear arrangement of the pseudo noise sources obtain similar results as the arc arrangement, with a difference in noise reduction of less than $1 \mathrm{~dB}$. A detailed comparison of the results is beyond the scope of this paper. In the simulations, the three pseudo noise sources are simultaneously driven by a tonal signal to mimic the noise from different directions.

For the primary noise that originates from a $60 \mathrm{~m}$ line of 13 random-phased incoherent point sources that is located $60 \mathrm{~m}$ from the ANC system, the effect of the distance from the pseudo noise sources to the ANC system on the performance is simulated and plotted against the dimensionless number $k y_{\mathrm{m}, 2} L_{0} / d_{0}$ in Fig. 7. In the simulations, the three-channel systems $(L=3, U=3)$ were investigated, and the second channel is on the $y$-axis, as shown in Fig. 6, where $y_{\mathrm{m}, 2}$ denotes the distance from the pseudo noise source to the ANC system. As a baseline for comparison, the simulation results for the fullycoupled three-channel system with three reference microphones are also shown in Fig. 7, where the vertical bars indicate the standard deviation of 100 trials.

As shown in Fig. 7, the average NR increases with distance from the pseudo noise sources to the ANC system and then approaches a constant, which is similar to the results for the single-channel system. The fully-coupled three-channel system with three reference microphones shows the highest $\mathrm{NR}$, as expected, which is approximately $3 \mathrm{~dB}$ higher than the three single-channel system when the pseudo noise sources are placed far from the ANC system $\left(k y_{\mathrm{m}, 2} L_{0} d_{0}>10 \pi\right)$. The performance of the three single-channel system is slightly superior to that of the coupled three-channel system with one reference microphone as the three single-channel systems have three reference microphones, which better detect noise from different directions. 


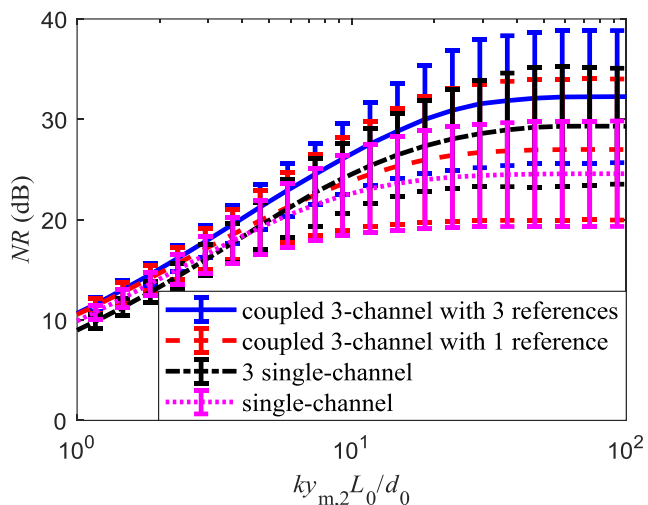

(a)

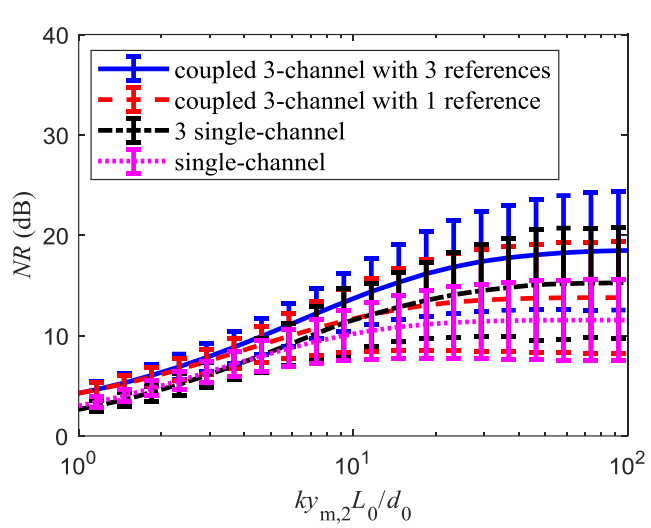

(c)

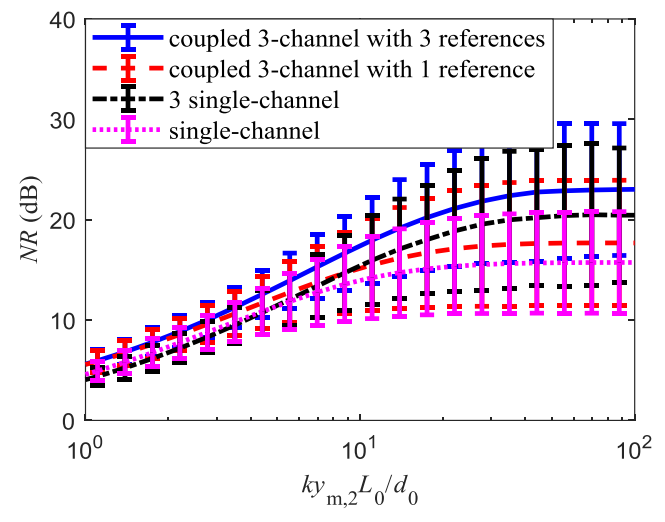

(b)

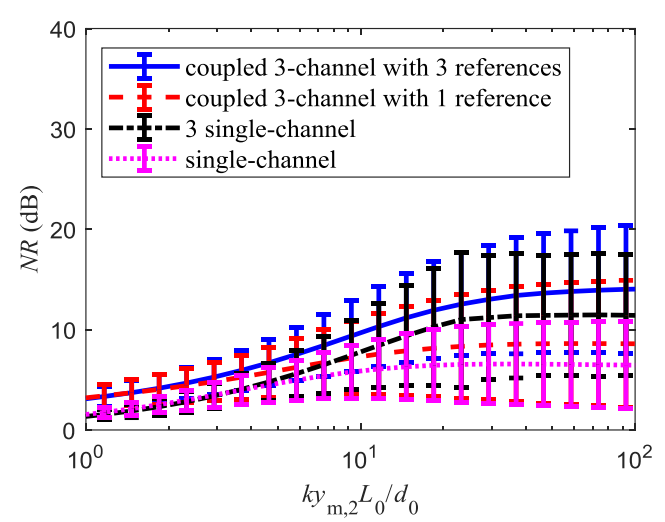

(d)

Fig. 7. NR (dB) as a function of the dimensionless number $k y_{\mathrm{m}, 2} L_{0} / d_{0}$ at (a) $100 \mathrm{~Hz}$, (b) $300 \mathrm{~Hz}$, (c) $500 \mathrm{~Hz}$ and (d) $1000 \mathrm{~Hz}$.

As shown in Fig. 7, the NR decreases with increasing frequency. For a clear illustration, the NR as a function of frequency is shown in Figs. 8(a) and 8(b) for the pseudo noise sources that are placed $1 \mathrm{~m}$ and $20 \mathrm{~m}$, respectively, from the ANC systems. As shown in Fig. 8, the performance of all systems decreases with increasing frequency and the three-channel system achieves a better performance than the single-channel ANC system. For primary noise from a $60 \mathrm{~m}$ line of 13 random-phased incoherent point sources that are located $60 \mathrm{~m}$ from the ANC system, the highest NR by the three single-channel system is approximately $30 \mathrm{~dB}$ at $100 \mathrm{~Hz}$ to $10 \mathrm{~dB}$ at $1000 \mathrm{~Hz}$, which can be achieved by placing the pseudo noise sources $20 \mathrm{~m}$ from the system. Note that the NR in Fig. 8 for the three-channel systems is slightly lower than that in Fig. 5 for the single-channel system as the NR in Fig. 8 is averaged over three error microphones while the NR in Fig. 5 is calculated for a single error microphone. 


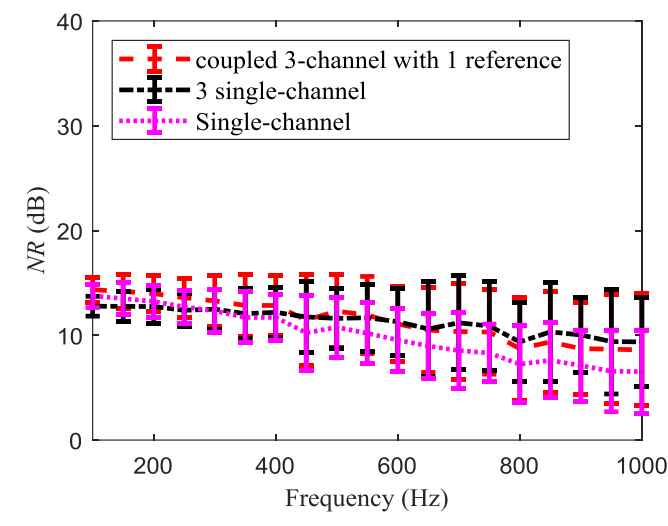

(a)

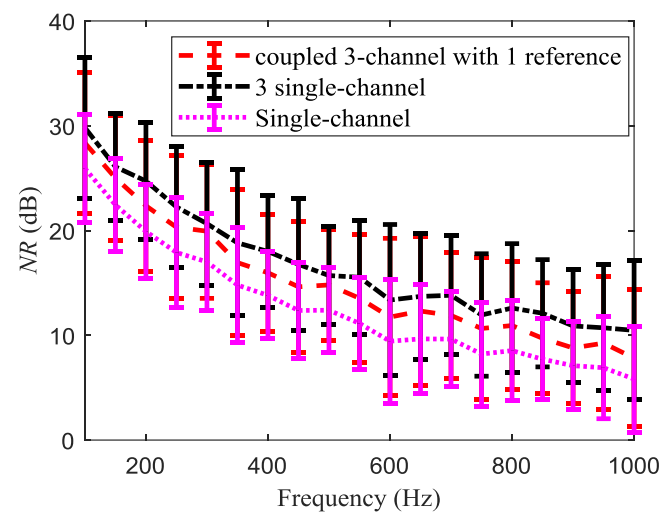

(b)

Fig. 8. NR $(\mathrm{dB})$ as a function of frequency for a distance between the pseudo noise sources and the

ANC system of (a) $y_{\mathrm{m}, 2}=1.0 \mathrm{~m}$ and (b) $y_{\mathrm{m}, 2}=20 \mathrm{~m}$.

The feasibility of the proposed pseudo noise source method is verified for the multiple singlechannel system and the coupled multichannel system with one reference microphone. Similar to the single-channel ANC system, the performance of the multichannel ANC systems can be improved by moving the pseudo noise sources farther from the ANC systems. The NR increases with the distance between the pseudo noise sources and the ANC system and then approaches a constant when the distance exceeds a critical value, which depends on frequency and the length of the far-field noise sources.

\section{Experiments}

Experiments for the single-channel ANC system were performed in a large open-plan room while the multichannel systems were tested in both a large open-plan room and outdoor environments. In the experiments, the reference microphones were Anty M1212U 1/2" unidirectional microphones, and the error microphones were Anty M1212 1/2" omnidirectional free-field microphones. An Anty MC08 eight-channel signal conditioner was used to connect the reference and error microphones to an Antysound Tiger ANC- II controller [29]. In the tuning stage, the secondary paths were modelled and then the controller coefficients were adjusted to cancel the pseudo noise sources. After optimal controller coefficients were determined for the pseudo noise sources, they were fixed and employed to cancel the far-field primary noise in the cancelling stage.

To model the secondary paths, a random noise signal was generated by the controller and played back through the control sources; the error signals were picked up by the error microphones and fed to the controller. An FIR filter was used to model the secondary paths from each control source to the error microphones. The step size of the FIR filters was adjusted to achieve a balance between the stability and the convergence speed. In the experiments, a step size of 0.01 and 0.1 were applied for the secondary path filters in the indoor experiments and outdoor experiments, respectively. 
After the secondary paths were modelled, the controller was used to cancel the pseudo noise signals. The controller generated a random noise signal, which was played back through the pseudo noise sources. The FxLMS algorithm was employed by the controller to adaptively adjust the control filter coefficients to minimize the error signals at the error microphones. The step size of the control filters was adjusted to achieve a balance between the stability and the convergence speed, and the leakage factor was adjusted to achieve a balance between the stability and the noise reduction. In the indoor experiments, the step size value and leakage factor were set to 0.01 and $10^{-6}$, respectively. In the outdoor experiments, the step size value and leakage factor were set to 0.1 and $10^{-4}$, respectively.

\subsection{Indoor single-channel ANC of tonal disturbance}

The experimental setup for the single-channel ANC system is shown in Fig. 9, where all cables are removed for clarity. The loudspeakers and microphones were placed on the ground to eliminate the reflections from the floor, and both the noise sources and the pseudo noise sources were placed within $1.0 \mathrm{~m}$ of the ANC system to ensure that the direct sound was dominant. In the indoor experiments, the Digitech CS-2478 loudspeakers served as control sources while the Genlec 6010 active loudspeakers served as primary and pseudo noise sources. In the experiments, the control source and error microphone were located $0.15 \mathrm{~m}$ and $0.3 \mathrm{~m}$, respectively, from the reference microphone. The reference microphone was located behind the control sources and is not shown in the photos (blocked by the control source).

Three noise sources were placed $1.0 \mathrm{~m}$ from the reference microphone to simulate the primary noise from different directions. In the first measurement, the single-channel ANC system was optimized with a single pseudo noise source, as shown in Fig. 9(a), and the pseudo noise source was removed and the system was used to cancel the noise from three primary noise sources. In the second measurement, three pseudo noise sources that mimic primary noise from different directions were employed to optimize the single-channel ANC system. After the single-channel ANC system was optimized, the three pseudo noise sources were removed and the system was used to cancel the noise from three primary noise sources, as shown in Fig. 9(b). 


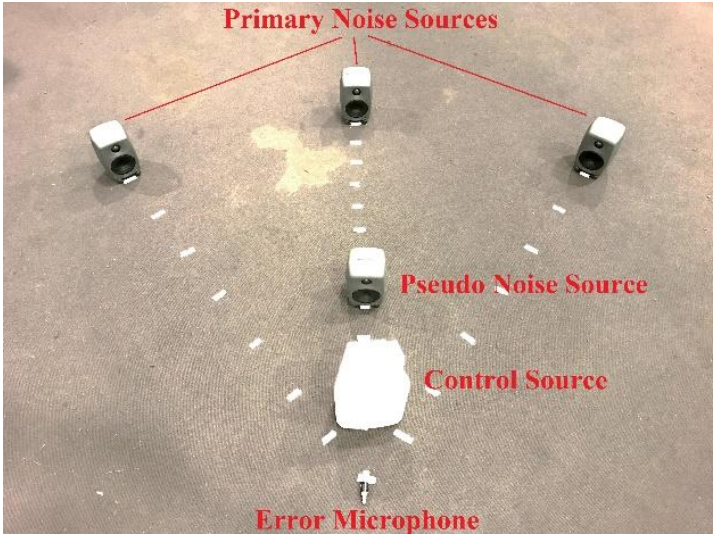

(a)

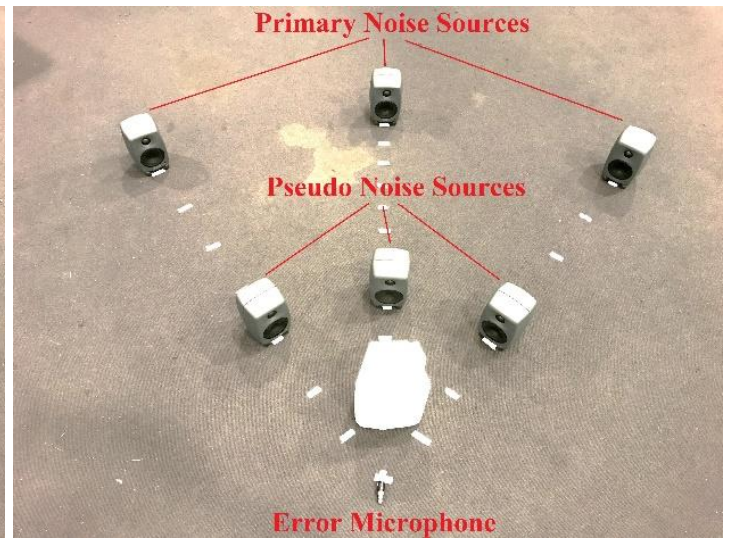

(b)

Fig. 9. Experimental setup for the single-channel ANC system with (a) a single pseudo noise source and (b) three pseudo noise sources in a large open-plan room.

The measurement results at $100 \mathrm{~Hz}$ and $300 \mathrm{~Hz}$ are compared with the simulation results in Fig. 10, where the simulation setup is the same as that in the measurements. Fig. 10 shows the NR as a function of distance between the pseudo noise sources and the single-channel ANC system. Moving the pseudo noise source farther from the ANC system increases the NR for both configurations, which in consistent with the simulation results. A comparison between Figs. 10(a) and 10(b) shows that the NR at $100 \mathrm{~Hz}$ is higher than that at $300 \mathrm{~Hz}$, which is consistent with the simulation results. In addition, using three pseudo noise sources can improve the performance compared with using a single pseudo noise source as more pseudo noise sources can better mimic the primary noise from different directions. The measurement results in Fig. 10 comply with the simulations and verify the conclusions obtained from the simulation results in Section 3.1.

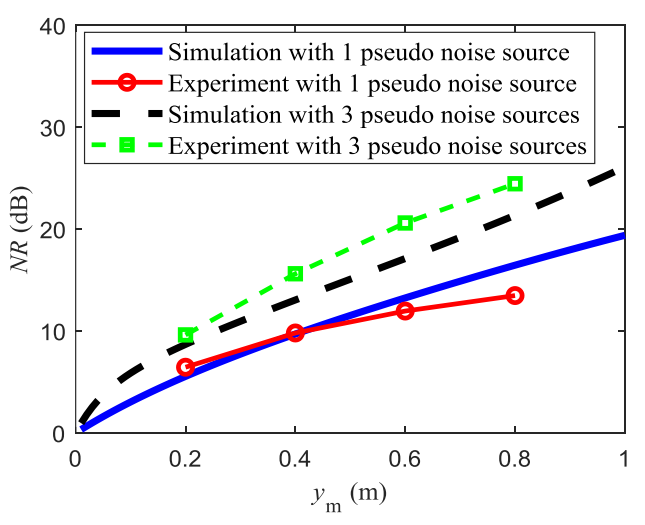

(a)

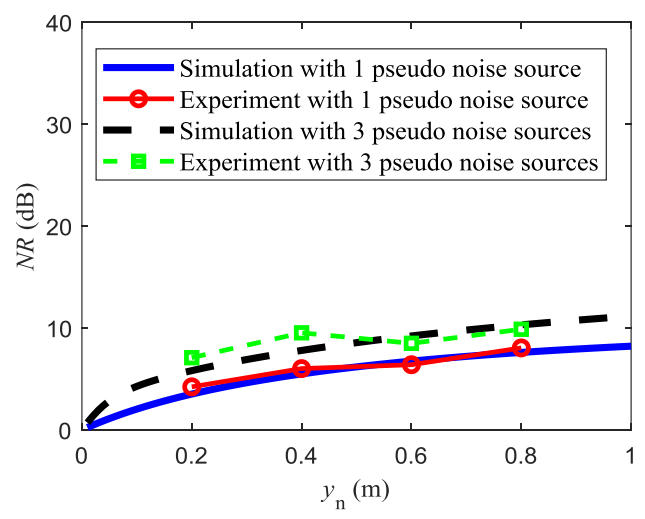

(b)

Fig. 10. Experimental results for the single-channel ANC system with three primary noise sources located $1.0 \mathrm{~m}$ from the system. NR (dB) as a function of the distance between the pseudo noise sources and the ANC system at (a) $100 \mathrm{~Hz}$ and (b) $300 \mathrm{~Hz}$. 


\subsection{Indoor multichannel ANC of tonal disturbance}

The performance of the three single-channel ANC system and the performance of the coupled threechannel system with one reference microphone were measured in a large open-plan room. The experimental setup for the three single-channel ANC system is shown in Fig. 11, where the reference microphones are blocked by the control sources. In the experimental setup in Fig. 11, the coordinates of the reference microphones, control sources and error microphones are the same as those shown in Table 1. The experimental setup for the coupled three-channel system with one reference microphone is the same as that in Fig. 11 and Table 1, with the exception that only one reference microphone at $(0$, $0,0)$ was employed. In the experiments, three pseudo noise sources were simultaneously active to mimic the noise from different directions. After the system was optimized, the three pseudo noise sources were removed and the system coefficients were fixed and applied to cancel the noise from three primary noise sources.

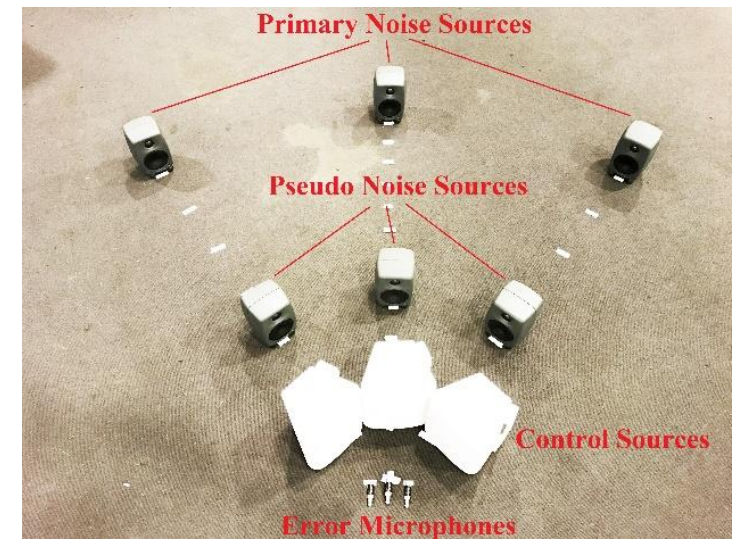

Fig. 11. Experimental setup for the three single-channel ANC system and the coupled threechannel ANC system with one reference microphone.

The measurement results for the three single-channel ANC system are compared with the simulation results in Fig. 12, where the simulation setup is equivalent to that in the measurements. In Fig. 12(a), the three primary noise sources were fixed $1.0 \mathrm{~m}$ from the system and the three pseudo noise sources were placed at different positions to study the effect on NR. For the noise sources far from the system, moving the pseudo noise sources farther away improves the performance, which shows agreement with the simulation results. In Fig. 12(b), the three pseudo noise sources were fixed $0.2 \mathrm{~m}$ from the system and the three noise sources were moved from $0.2 \mathrm{~m}$ from the system to $1.0 \mathrm{~m}$ from the system to study the effect of the primary noise source locations on the system NR performance. Figure 12(b) shows that the system performance is optimal when the positions of the pseudo noise sources are identical to the positions of the primary noise sources, and the NR decreases with the distance between the primary noise sources and the system. 
Note that the measured $\mathrm{NR}$ at $300 \mathrm{~Hz}$ is approximately $3 \mathrm{~dB}$ lower than that at $100 \mathrm{~Hz}$ due to the measurement uncertainties. In the experiments, noise sources were placed near the ANC system to render the direct sound dominant and the reflections from the walls and ceiling negligible. However, some reflections and scattering occurred from nearby tables and chairs. Although the pseudo noise sources and primary noise sources were placed at the labelled positions in Fig. 11, the acoustic centre may slightly differ for the measurements at $100 \mathrm{~Hz}$ and $300 \mathrm{~Hz}$. The noise reduction measured at 300 $\mathrm{Hz}$ may not be lower than that at $100 \mathrm{~Hz}$, as shown in Fig. 12(b).

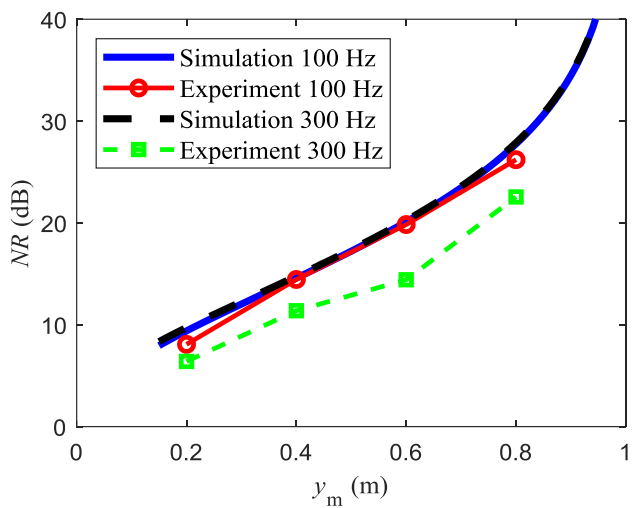

(a)

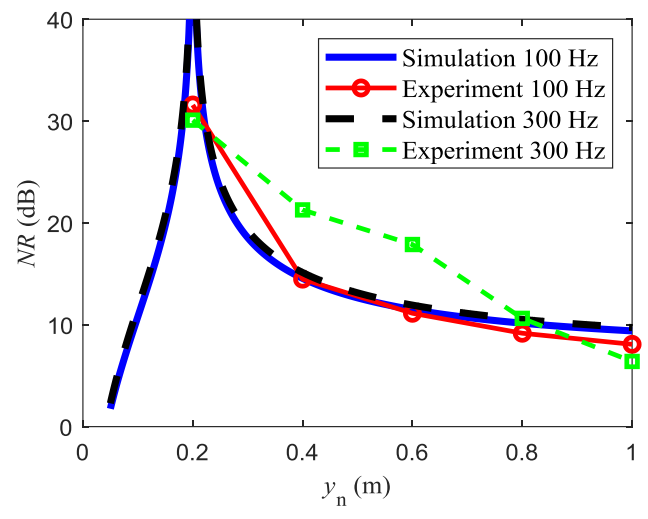

(b)
475

476

477

Fig. 12. Experimental results for the three single-channel ANC system. (a) NR in dB as a function of distance from the pseudo noise sources to the ANC system when the primary noise sources are located $1.0 \mathrm{~m}$ from the system. (b) NR in $\mathrm{dB}$ as a function of distance from the primary noise source to the ANC system when pseudo noise sources are located $0.2 \mathrm{~m}$ from the system.

Similarly, the measurement results for the coupled three-channel ANC system with one reference microphone are compared with the simulation results in Fig. 13, where the simulation setup is the same as that in the measurements. Moving the pseudo noise sources farther away increases the NR for farfield noise sources, which shows agreement with the simulation results. When the noise sources and pseudo noise sources are located $1.0 \mathrm{~m}$ from the ANC system and $0.2 \mathrm{~m}$ from the ANC system, respectively, the measured NR in Fig. 13(b) is approximately $3 \mathrm{~dB}$ higher than that in Fig. 13(a). This finding might be attributed to the measurement uncertainties, e.g., the positions of the noise sources and pseudo noise sources were not identical in the two measurements. The consistency between the simulation results and the measurement results in Figs. 12 and 13 demonstrates the feasibility of the proposed pseudo noise source method for tuning the ANC system to far-field noise control. 


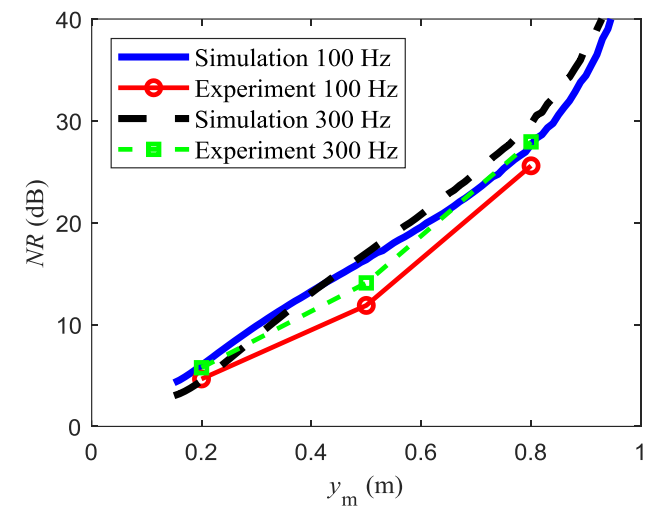

(a)

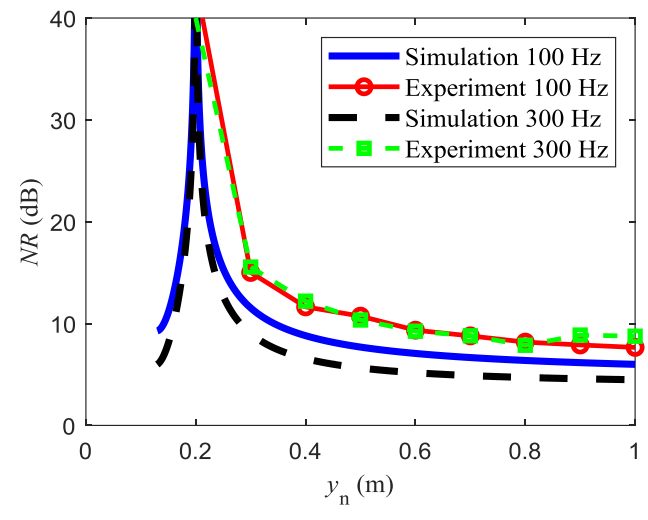

(b)

Fig. 13. Experimental results for the coupled three-channel ANC system with only one reference microphone. (a) NR in $\mathrm{dB}$ as a function of distance from the pseudo noise sources to the ANC system when the primary noise sources are located $1.0 \mathrm{~m}$ from the system. (b) NR in $\mathrm{dB}$ as a function of distance from the primary noise source to the ANC system when the pseudo noise sources are located $0.2 \mathrm{~m}$ from the system.

\subsection{Outdoor multichannel ANC of broadband disturbance}

To further evaluate the performance of the proposed method in real applications, an outdoor experiment was conducted in Richmond, Victoria, Australia. The experimental setup is shown in Fig. 14(a), where a six-channel system with one reference microphone was employed in the experiment. As illustrated in Fig. 14(a), the six control sources were placed along an arc to create a small quiet zone. The reference microphone was placed $1.6 \mathrm{~m}$ from the control sources to reduce the effect of acoustic feedback from the control sources for better ANC system stability and to ensure the causality of the ANC system, which has an inherent delay of $375 \mu$ s due to the AD/DA converters and digital signal processing. A pseudo noise source was placed $20 \mathrm{~cm}$ in front of the reference microphone. The purpose of the outdoor experiments was to demonstrate the use of a virtual sound barrier to create a small quiet zone behind the array of control sources, as shown in Fig. 14(a). When the outdoor experiments were conducted, only one loudspeaker was available to act as the pseudo noise source, which was placed in the direction of traffic noise to tune the controller.

In the tuning stage, random white noise below $500 \mathrm{~Hz}$ was produced through the pseudo noise source loudspeaker to adjust the control filter coefficients. After the coefficients were optimized, the pseudo noise source was removed and the system coefficients were fixed to cancel the traffic noise from the motorway that was located approximately $60 \mathrm{~m}$ from the ANC system. Although the simulation results to $1000 \mathrm{~Hz}$ are shown in Section 3, low frequencies below $500 \mathrm{~Hz}$ was the principal interest for outdoor traffic noise, especially noise caused by heavy trucks. Therefore, the ANC system was trained only for frequencies below $500 \mathrm{~Hz}$ in the outdoor experiments. 
In the measurements, the traffic noise was recorded by a Neumann KU100 dummy head behind the ANC system (not shown in this paper) for three minutes with a sampling rate of $48 \mathrm{kHz}$ when the ANC system was both on and off, and the Welch method was applied to estimate the power spectral density with a window size of 8192 samples and 50\% overlap. The results, which are shown in Fig. 14(b), reveals that a maximum NR of $9 \mathrm{~dB}$ is achieved below $400 \mathrm{~Hz}$ but noise above $400 \mathrm{~Hz}$ is not reduced. The total NR in the frequency range below $500 \mathrm{~Hz}$ is $3 \mathrm{~dB}$. The measured NR is not as acceptable as that from the simulations, which exceeds $20 \mathrm{~dB}$ below $400 \mathrm{~Hz}$ as the simulations are performed for tonal sound signals while the measured noise is broadband, and only one pseudo noise source was used in the experiments due to a limitation of available equipment. In future studies, multiple pseudo noise sources will be utilized to imitate traffic noise from different directions.

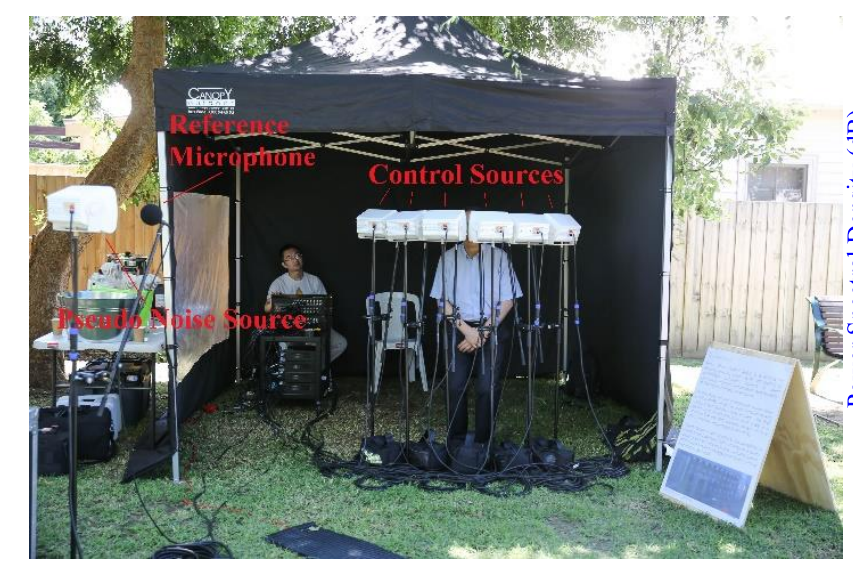

(a)

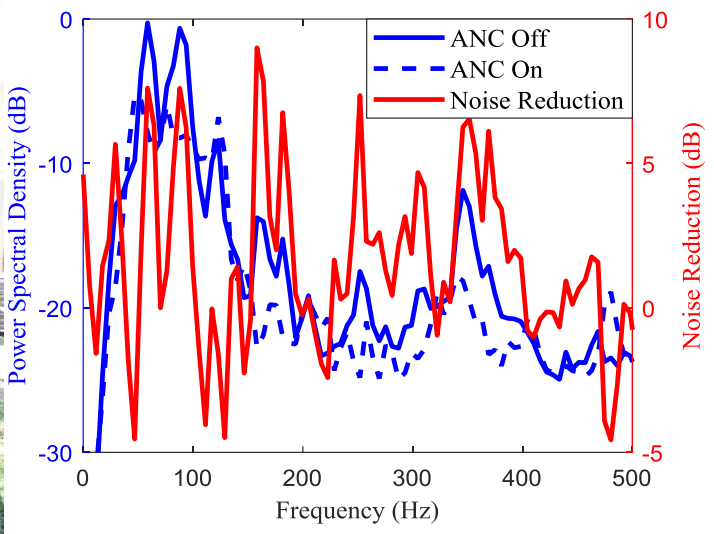

(b)

Fig. 14. (a) Experimental setup and (b) measurement results for the six-channel ANC system with only one reference microphone in outdoor environments.

Both indoor and outdoor experiments were conducted to investigate the performance of the proposed pseudo noise source method for configuring the fixed-coefficients feedforward active noise control system. The measurement results of the single-channel system, the multiple single-channel system, and the coupled multichannel system with one reference microphone are consistent with the simulation results, which validates the feasibility of the proposed method.

\section{Discussions}

The limitations of the current study and directions for future research are discussed in this section. In the theoretical analysis in Section 2 and the simulations in Section 3, the regularization factor was assumed to be zero after Eq. (6) for the best performance. Although the ill-conditioning problem was not encountered in the simulations in Section 3, a non-zero regularization factor is needed to increase the stability of the ANC system for non-stationary traffic noise signals at the cost of reducing the noise 
reduction performance. Therefore, the simulation results provide a reference for the upper limit performance, which can be achieved by the proposed method, rather than a reference for the final performance for real applications in the future.

The simulation results show the noise reduction performance at the error microphones without measuring the size of the quiet zone, which depends on many factors, such as frequency, control source locations, and error microphone positions. A detailed analysis of the effect of these factors on the quiet zone is provided in the literature [14,30], which is beyond the scope of this paper. The contribution of this paper is the proposed pseudo noise source method for configuring the active noise control system for traffic noise. The noise reduction at the error microphone usually has the highest value, which is a suitable measure for examining the feasibility of the proposed method. Therefore, it is employed to show the simulation results in this paper.

In the indoor experiments, both the primary noise sources and the pseudo noise sources were placed near the ANC system to make the direct sound dominant, which enables the reflections from the walls and ceiling to be disregarded. The primary noise is not considered as the far-field sound. One contribution of the proposed method is that the ANC system can achieve a noise reduction when the pseudo noise sources are placed at different locations from the primary noise sources. Due to the limitations of indoor experiments, the specific values of the NR cannot act as a reference for outdoor traffic noise control. However, the indoor experiments can be used to demonstrate the feasibility of the proposed method to tune the ANC system using pseudo noise sources and verify the simulation scheme. Future research can include indoor experiments in an anechoic chamber, in which the system is set up as close to the practical outdoor applications as possible.

In the outdoor experiments, only one loudspeaker was available for use as the pseudo noise source when the experiment was conducted, and the pseudo noise source was placed near $(20 \mathrm{~cm})$ the reference microphone to ensure that the pseudo noise source signal is considerably higher than the background noise. As the experiments were performed outdoors, the traffic noise from the motorway is loud. If the pseudo noise source was placed far from the reference microphone, the pseudo noise sources signal would be masked by the traffic noise. The usage of only one pseudo noise source near the reference microphone may cause inferior performance in the measurements. In practical applications in the future, additional pseudo noise sources will be needed to mimic the far-field traffic noise and achieve better performance.

In Ref. [22], a loudspeaker was mounted on a car that operated at $30 \mathrm{~km} / \mathrm{h}$ in outdoor experiments, where approximately 5-9 dB of noise reduction was achieved at the error microphones. In Ref. [23], a loudspeaker was mounted on a traversing system in an anechoic chamber to mimic a moving sound source, and noise reduction at the error microphone was approximately $10 \mathrm{~dB}$ between $100 \mathrm{~Hz}$ and 200 $\mathrm{Hz}$ for a moving speed of $1 \mathrm{~m} / \mathrm{s}$. However, no noise reduction occurred below $100 \mathrm{~Hz}$ or above $200 \mathrm{~Hz}$ [23]. These studies focused on active control of noise from a single moving primary noise source. In practice, multiple moving noise sources often exist in traffic noise, which may worsen the performance. 
This study uses a different approach by modelling the traffic noise as a line of incoherent point sources in the simulations, which is similar to realistic motorway noise. In the outdoor experiments of this research, the noise reduction was evaluated by a Neumann KU100 dummy head instead of the measurement at the error microphones. Therefore, obtaining a clear conclusion by directly comparing the results of this research with that of [22] and [23] is difficult.

\section{Conclusions}

This study proposed a pseudo noise source method for configuring fixed-coefficient ANC systems for traffic noise control. Numerical simulations were performed for both a single-channel ANC system and a multichannel ANC system to study the noise reduction performance of the proposed method for a long line of incoherent noise sources located $60 \mathrm{~m}$ from the system. The findings indicated that the noise reduction increased with the distance between the pseudo noise sources and the ANC system and then approached a constant when the distance exceeded a critical value, i.e., when the dimensionless number $k y_{\mathrm{m}} L_{0} / d_{0}$ was larger than $10 \pi$. Experiments with a single-channel ANC system, a multichannel ANC system with three single-channel modules, and a coupled three-channel ANC system with one reference microphone were conducted in a large open-plan room to control the noise from three farfield noise sources. The measurement results agreed with the simulation results, in general, demonstrating the feasibility of the proposed method. An outdoor onsite experiment was also conducted with a coupled six-channel ANC system with one reference microphone to further verify the proposed method. Two limitations of this study are that acoustic feedback from the control sources to the reference microphones was not considered and the system was not adaptive. Future research will explore the proposed method of multiple single-channel ANC systems with practical applications for outdoor traffic noise control to create a large quiet area.

\section{Acknowledgements}

This research was supported by the Transurban Innovation Grants program and the Australian Research Council's Linkage Projects funding scheme (LP140100987). The authors are grateful to the editor and two anonymous reviewers for their constructive suggestions to improve the quality of the manuscript.

\section{References}

[1] Guo J, Pan J, Hodgson M. A brief review of active control of environmental noise and its applications. Proceddings 14th Int. Congr. Sound Vib., 2007, p. 1-8.

[2] Avsar Y, Gonullu MT. Determination of safe distance between roadway and school buildings to get acceptable school outdoor noise level by using noise barriers. Build Environ 2005;40:1255-60. doi:10.1016/j.buildenv.2004.10.020. 
[3] Zhao S, Qiu X, Cheng J. An integral equation method for calculating sound field diffracted by a rigid barrier on an impedance ground. J Acoust Soc Am 2015;138:1608-13. doi:10.1121/1.4929933.

[4] Cianfrini C, Corcione M, Fontana L. Experimental verification of the acoustic performance of diffusive roadside noise barriers. Appl Acoust 2007;68:1357-72. doi:10.1016/j.apacoust.2006.07.018.

Asdrubali F, Pispola G. Properties of transparent sound-absorbing panels for use in noise

[6] Ishizuka T, Fujiwara K. Performance of noise barriers with various edge shapes and acoustical conditions. Appl Acoust 2004;65:125-41. doi:10.1016/j.apacoust.2003.08.006.

Yang C, Pan J, Cheng L. A mechanism study of sound wave-trapping barriers. J Acoust Soc Am 2013;134:1960-9. doi:10.1121/1.4921279.

[12] Guo J, Pan J. Actively created quiet zones for broadband noise using multiple control sources and error microphones. J Acoust Soc Am 1999;105:2294-303. doi:10.1121/1.426836.

[14] Zou H, Qiu X, Lu J, Niu F. A preliminary experimental study on virtual sound barrier system. J Sound Vib 2007;307:379-85. doi:10.1016/j.jsv.2007.06.042.

[15] Epain N, Friot E. Active control of sound inside a sphere via control of the acoustic pressure at product-type active soft edge noise barrier. Proc. 18th Int. Congr. Acoust., Kyoto, Japan: 2004, p. 1041-4.

[17] Elliot SJ. Signal Processing for Active Control. vol. 23. Academic Press; 1996.

[18] Zou H, Lu J, Qiu X. The active noise barrier with decentralized feedforward control system. Proc. 17th Int. Congr. Sound Vib., Cairo: 2010, p. 18-22.

[19] Qiu X. Recent advances on active control of sound transmission through ventilation windows. 
Proc. 24th Int. Congr. Sound Vib., London: 2013, p. 1-8.

[20] Murao T, Nishimura M, He J, Lam B, Ranjan R. Feasibility study on decentralized control system for active acoustic shielding. Proc. INTER-NOISE 2016, Hamburg: 2016, p. 462-71.

[21] Murao T, Nishimura M, Sakurama K, Nishida S. Basic study on active acoustic shielding (Improving the method to enlarge the AAS window). Mech Eng J 2016;3:1-12. doi:10.1299/mej.15-00322.

[22] Uesaka K, Ohnishi H, Hachimine K, Nishimura M, Ohnish K. Active control of sound from a moving noise source. Proc. 1997 Symp. Act. Control Sound Vib., Budapest, Hungary: 1997, p. 1125-34.

[23] Omoto A, Matsui T, Fujiwara K. Behaviour of an adaptive algorithm with a moving primary source. J Acoust Soc Japan 1998;19:211-21.

[24] Lacey J, Harvey L, Moore S, Qiu X, Pink S, Zhao S, et al. Soundscape design of motorway parkland environments - transformation, cancellation, and ethnography. Proc. Invis. Places 2017, Azores, Portugal: 2017, p. 52-63.

[25] Tu Y. Multiple Reference Active Noise Control. Virginia Polytechinic Institute, 1997.

[26] Tao J, Wang S, Qiu X, Pan J. Performance of an independent planar virtual sound barrier at the opening of a rectangular enclosure. Appl Acoust 2016;105:215-23. doi:10.1016/j.apacoust.2015.12.019.

[27] Hansen CH, Snyder SD, Qiu X, Brooks L, Moreau D. Active Control of Noise and Vibration. 2nd ed. CRC Press; 2012.

[28] Steele C. A critical review of some traffic noise prediction models. Appl Acoust 2001;62:27187. doi:10.1016/S0003-682X(00)00030-X.

[29] Antysound. Tiger ANC-II n.d. http://www.antysound.com/ (accessed September 8, 2018).

[30] Zou H, Qiu X. Performance analysis of the virtual sound barrier system with a diffracting sphere. Appl Acoust 2008;69:875-83. doi:10.1016/j.apacoust.2007.06.002. 
688

689

690

691

692

693

694

695

696

697

698

699

700

701

702

703

704

705

706

707

708

709

710

711

712

713

714

715

716

717

718

719

\section{Appendix}

The NR dependency on distance and frequency is formulated for the single-channel ANC system. For a single-channel ANC system, the total sound pressure at the error microphone can be written as

$$
e(\omega)=p(\omega)+q(\omega) x(\omega) Z_{\mathrm{ce}}(\omega),
$$

where $p(\omega)$ is the primary noise pressure at the error microphone, $x(\omega)$ is the sound pressure at the reference microphone, $q(\omega)$ represents the controller coefficients, and $Z_{\mathrm{ce}}(\omega)$ is the transfer function from the control source to the error microphone. By setting the cost function as the squared sound pressure at the error microphone, the optimal controller response can be obtained as

$$
q(\omega)=-\frac{p(\omega)}{x(\omega) Z_{\mathrm{ce}}(\omega)}
$$

For the proposed pseudo noise source scheme, the controller is optimized for the pseudo noise source placed at $\mathbf{r}_{\mathrm{m}}$ in front of the ANC system; thus,

$$
q_{\mathrm{m}}(\omega)=-\frac{p_{\mathrm{m}}(\omega)}{x_{\mathrm{m}}(\omega) Z_{\mathrm{ce}}(\omega)},
$$

where $x_{\mathrm{m}}(\omega)$ and $p_{\mathrm{m}}(\omega)$ are the sound pressure due to the pseudo noise source at the reference microphone and the error microphone, respectively. This optimized controller is utilized to control the primary noise source in the far field. Substituting Eq. (A.3) into Eq. (A.1), the sound pressure at the error microphone can be obtained as

$$
e(\omega)=p(\omega)-\frac{x(\omega)}{x_{\mathrm{m}}(\omega)} p_{\mathrm{m}}(\omega) .
$$

Therefore, the noise reduction $(\mathrm{dB})$ at the error microphone can be derived as

$$
N R(\omega)=10 \log _{10}\left(\left|\frac{p(\omega)}{p(\omega)-\frac{x(\omega)}{x_{\mathrm{m}}(\omega)} p_{\mathrm{m}}(\omega)}\right|^{2}\right) .
$$

Eq. (A.5) shows that the noise reduction performance of the single-channel system is determined by the sound pressure at the error microphone and the reference microphone due to the actual noise source and the pseudo noise source, respectively.

If only one pseudo noise source is present in the free field, the sound pressure due to the pseudo noise source at the reference microphone and error microphone are

$$
x_{\mathrm{m}}(\omega)=A_{\mathrm{m}} \frac{e^{-\mathrm{j} k R_{\mathrm{mr}}}}{4 \pi R_{\mathrm{mr}}},
$$

and

$$
p_{\mathrm{m}}(\omega)=A_{\mathrm{m}} \frac{e^{-\mathrm{j} k R_{\mathrm{me}}}}{4 \pi R_{\mathrm{me}}},
$$

respectively, where $A_{\mathrm{m}}$ is the amplitude of the pseudo noise source, $k$ is the wavenumber, $\mathrm{j}$ is the imaginary unit, and $R_{\mathrm{mr}}=\left|\mathbf{r}_{\mathrm{m}}-\mathbf{r}_{\mathrm{r}}\right|$ and $R_{\mathrm{me}}=\left|\mathbf{r}_{\mathrm{m}}-\mathbf{r}_{\mathrm{e}}\right|$ are the distance from the pseudo noise source to the reference microphone and the error microphone, respectively. Similarly, the sound pressure due to the actual noise source at the reference microphone and error microphone are

$$
x(\omega)=A_{\mathrm{n}} \frac{e^{-\mathrm{j} k R_{\mathrm{nr}}}}{4 \pi R_{\mathrm{nr}}},
$$


and

$$
p(\omega)=A_{\mathrm{n}} \frac{e^{-\mathrm{j} k R_{\mathrm{ne}}}}{4 \pi R_{\mathrm{ne}}}
$$

722 respectively, where $A_{\mathrm{n}}$ is the amplitude of the noise source and $R_{\mathrm{nr}}=\left|\mathbf{r}_{\mathrm{n}}-\mathbf{r}_{\mathrm{r}}\right|$ and $R_{\mathrm{ne}}=\left|\mathbf{r}_{\mathrm{n}}-\mathbf{r}_{\mathrm{e}}\right|$ are the 723 distance from the noise source at $\mathbf{r}_{\mathrm{n}}$ to the reference microphone and error microphone, respectively.

724 Substituting Eqs. (A.6) and (A.7) into Eq. (A.5),

$$
N R(\omega)=-10 \log _{10}\left(\left|1-\frac{R_{\mathrm{ne}}}{R_{\mathrm{nr}}} \frac{R_{\mathrm{mr}}}{R_{\mathrm{me}}} e^{-\mathrm{j} k\left(R_{\mathrm{nr}}-R_{\mathrm{ne}}+R_{\mathrm{me}}-R_{\mathrm{mr}}\right)}\right|^{2}\right) .
$$

If both the noise source and the pseudo noise source are on the $y$ axis, as shown in Fig. 1(a), $R_{\mathrm{ne}}=$ $R_{\mathrm{nr}}+d$ and $R_{\mathrm{me}}=R_{\mathrm{mr}}+d(d$ is the distance between the reference microphone and error microphone), the NR is independent of frequency as the exponential term in Eq. (A.8) is 0 . If the distance from the noise source to the reference microphone $R_{\mathrm{nr}}$ is considerably larger than $d$, then $R_{\mathrm{ne}} \approx R_{\mathrm{nr}}$, Eq. (A.8) can be simplified as

$$
N R(\omega)=-10 \log _{10}\left(\left|1-\frac{R_{\mathrm{mr}}}{R_{\mathrm{mr}}+d}\right|^{2}\right) .
$$

732 If the pseudo noise source is far from the ANC system, i.e., $R_{\mathrm{mr}}$ is considerably larger than $d$, then the

733 NR can be further simplified as

$$
N R(\omega)=20 \log _{10}\left(\frac{R_{\mathrm{mr}}}{d}\right) .
$$

Eq. (A10) shows that the NR for a far-field noise source increases by $6 \mathrm{~dB}$ for a fixed ANC system when the distance between the pseudo noise source and the reference microphone doubles, but is independent of the location of the real primary noise source. This result is different from the results in Figs. 3 and 7, where NR increases with distance and then approaches a constant when the distance exceeds a critical value, as Eqs. (A.8) to (A.10) are only valid when only one primary noise source and only one pseudo noise source are utilized. When multiple primary noise sources exist, as in this study, the noise reduction increases with distance and then approaches a constant when the distance exceeds a critical value, i.e., $k y_{\mathrm{m}} L_{0} / d_{0}>10 \pi$. 